\title{
TITLE:
}

\section{PARASITIC GASTROPODS FOUND IN ECHINODERMS FROM JAPAN}

AUTHOR(S):

Habe, Tadashige

CITATION:

Habe, Tadashige. PARASITIC GASTROPODS FOUND IN ECHINODERMS FROM JAPAN. PUBLICATIONS OF THE SETO MARINE BIOLOGICAL LABORATORY 1952, 2(2): 73-85

ISSUE DATE:

1952-10-05

URL:

http://hdl.handle.net/2433/174685

RIGHT: 


\title{
PARASITIC GASTROPODS FOUND IN ECHINODEFMS FROM JAPAN ${ }^{*}$
}

\author{
TADASHige HABE \\ Zoological Iustitute, Kyoto University
}

With Plate VI

Hitherto twenty one species of gastropods parasitic on echinoderms have been recorded from Japan by various authors, such as RANDALL et HEATH (1912), S. Hirase $(1920,1927,1932)$, Dall (1925), GislEN (1927), Is. TAKI (1929), IWANOFF (1933), MORTENSEN (1940, 1943), KAWAHARA (1943), HABE (1944, 1950, 1951), Kuroda (1949) and Kuroda et HABE (1950). In this paper eight more species are added to this list. Of these six are new to science and also parasitic habits are confirmed in other two species which have never been noticed in this country.

It is my pleasant duty to acknowledge here my deep indebtedness to Dr. Taku KomaI and Dr. Tokubei Kuroda for their kind direction and encouragement in the course of my study. My hearty thanks are also due to Prof. Denzaburo MiYADI, Dr. Iwao TAKI, Dr. Huzio Utinomi, Dr. Takasi Tokioka, Messrs. Toshihiko Yamanout, Torao Yamamoto, Akibumi Teramachi, Masuoki HorIKosI and Takashi SAITo for their kindness in placing their collections at my disposal.

\section{Family EulimidaE \\ Genus Balcis LFACH 1847}

1. Balcis kuronamako new species

(P1. VI, Fig. 20)

Shell subulate, slightly bending backwardly on the early whorls, rather solid, milky white except for the snowy white early whorls, smooth and polished; apex rather small but blunt; whorls 11 in number, each whorl rather

* Contributions from the Seto Marine Biological Laboratory, No. 178.

Publ. Seto Mar. Biol. Lab., II (2), 1952. (Article 6) 
flattened; suture appressed; body whorl two-fifths of the shell length, gently arcuate on the base; varices irregularly disposed on the right side; aperture ovate; outer margin arcuate forwardly; columellar margin thickened and rather straightened; umbilicus closed. Operculum thin, horny, paucispiral as in other eulimids.

Length $8.3 \mathrm{~mm}$, breadth $2.6 \mathrm{~mm}$ (figured type specimen).

Habit: On the surface of Holothuria aff. vagabunda SelenKa-blackcoloured littoral sea-cucumber.

Type locality: Shirahama, Wakayama Pref., Honshu.

Di tribution: The coast of Wakayama Pref., Honshu (Shirahama, Fukuro, Mirotsu and Satono).

Remarks: Balcis aciculatus (PEASE 1860)1,2 from Hawaii and Balcis venustus (PEASE 1867) ${ }^{3}$ from Tahiti are very much allied to this new species. However, both are smaller even in the adult stage and unknown about the parasitic features. Balcis soliduloides HABE 1951 is also smaller in size and stouter than the present new species.

\section{Balcis soliduloides HABE}

(P1. VI, Fig. 25)

1943 Fulima sp. KAWAHARA, Botany and Zoology (Japan), 11, p. 976, figs. 3B, C.

1951 Balcis solidu'ờd HABE, Illust. Cat. Jap. Shells, No. 14, p. 94.

Length $7.5 \mathrm{~mm}$, breadth $2.8 \mathrm{~mm}$ (figure 1 type specimen).

Habit: On the surface of Holothuria atra JAEGER, Ho'othuria argus (JAEGER) and Stichopus chloronotus BRANDT.

Distribution: Suô, Formosa (type locality) and Ishigaki-jima.

\section{Balcis yamazii new species}

(Pl. VI, Figs. 6, 9)

Shell acutely pyramidal, somewhat bent to the right side, milky white except for the snowy early whorls, rather solid, smooth and polished; whorls 8 in number; each whorl slightly convex and suture impressed distinctly; all varices decidedly impressed on the right side of each whorl and form a slowly receding ascending line; body whorl one-third of shell length, of which the

1) Proc. Zool. Soc. London, 1860, p. 438.

2) PIisbry 1917, Proc. Acad. Nat. Sci. Phila., 69, p. 222, figs. 11a, b, pl. 14, fig. 8.

3) Amer. Jour. Conch., 3, p. 294, pl. 24, fig. 24. 
periphery obtusely angulated and the base rather flattened; aperture rather small, ovate; outer margin roundly curved, somewhat thickened and forwardly arcuate, columellar margin short, curved and thickened; umbilical fissure present on the outside of it.

Length $7.5 \mathrm{~mm}$, breadth $3.1 \mathrm{~mm}$ (figured type specimen).

Habit: On the surface of Holothuria lubrica var. mocbii (LUDWIG).

Type locality: Shirahama, Wakayama Pref, Honshu.

Remarks: Balcis bovicornus (PIISBRY 1905) ${ }^{4}$ from Ogasawara Islands has the quite round body whorl. Balcis tortuosus (A. AdAMs et ReEve 1850) ${ }^{5}$ from the China Sea is another allied species, but it has slenderer and more distinctly tortuous form. Balcis kanaka (PILSBRY 1917) ${ }^{6}$ from Hawaii is a robuster species.

\section{Balcis astropectenicola KURODA et HABE}

(P1. VI, Fig. 16)

1950 Balcis astropsctenicola KuRODA et HABI, Tllust. Cat. Jap. Shells, No. 9, p. 60.

Length $7.2 \mathrm{~mm}$, breadth $2.4 \mathrm{~mm}$ (figured type specimen).

Habit: On the oral side of Astropecten polyacanthus Múleter et TroscheL. and Luidia maculata Múlter et Troschel-littoral sea-stars.

Distribution: Tanabe Bay, Wakayama Pref. (type locality) and Miho Bay, Tottori Pref., Honshu.

\section{Balcis robusius (A. ADAMS)}

(P1. VI, Fig. 4)

1861 Fulima robusta A. ADAMS, Ann. Mag. Nat. Hist。 (3) 7 (38), p. 125.

1886 Eulima robusta TRYON, Man. Conch., 8, p. 271, pl. 68, fig. 25.

1895 Eulima robusta PILSBRX, Cat. Mar. Moll. Japan, p. 77.

Length $4.8 \mathrm{~mm}$, breadth $2.4 \mathrm{~mm}$ (figured specimen collected from Ago Bay, Mie Pref., Honshu).

Habit: On the oral side of Astropecten polyacanthus Múller et TROSCHEL - iittoral sea-star.

4) Proc. Acad. Nat. Sci. Phila, 57, p. 111, pl. 11, figs. 9, 10.

5) Zool. Voy. Samarang, Moll., p. 53, pl. 11, fig. 26.

6) Proc. Acad. Nat. Sci. Phila., 69, p. 310, fig. 3. 
Distribution: Mishima (=Mino-shima), in the Japan Sea (type locality); Shirahama, Wakayama Pref. and Ago Bay, Mie Pref., Honshu.

\section{Balcis acicula (GoULD)}

1849 Stilifer acicula Goul.D, Proc. Boston Soc. Nat. Hist., 3, p. 84.

1854 Eulima vitrea A. A.DAMS, Proc. Zool. Soc. London, (1851), p. 277.

1854 Eulima vitrea A. ADAMS, Thes. Conch., 2, p. 799, pl. 169, fig. 35.

1862 Stilifer acicula Gould, Otia Conch., pp. 54, 245.

1865 Eulima vitrea SOWERBY, Conch. Icon., p. 19.

1886 Eulima acicula TRYON, Man. Conch., 8, p. 282, pl. 70, figs. 92-95.

1899 Eulima vitrea CleSsIN, Conch. Cab.. 1 (28), p. 10, pl. 2 , fig. 7.

1903 Eulima vitrea SCHEPMAN, Siboga Exped., monogr. 49c, p. 235.

1917. Melanella acicula PILSBRY, Proc. Acad. Nat. Sci. Phila., 69, p. 223.

1943 Museronalia sp. KAWAHARA, Botany and Zoology (Japani, 11 (12), p. 976, fig. 3A.

Shell straight, slender, elongate, thin, transparent, smooth and highly polished; apex small, milky white: whorls 12 or 13; each whorl flattened; suture weakly appressed and coloured with lucid white; arerture elongate oval; columellar margin dilated and thickened; outer margin strongly arched forwardly; operculated.

Length $8.7 \mathrm{~mm}$, breadth $2.2 \mathrm{~mm}$ (examined specimen collected from Palao).

Habit: In the coelom or on the surface of Stichopus chloronotus BRANDT.

Distribution: Fejee Islands (type locality); Hawaii Islands; Palao; Ishigaki Island, Loochoo.

\section{Balcis teramachii new species}

(P1. VI, Figs. 26, 27)

Shell straight, smooth, glossy and semitransparently milky white; whorls 15 in number, each whorl flattened and suture weakly appressed; body whorl about one-third of the shell length, of which the base is rounded; aperture ovate; outer margin roundly arched and arcuate forwardly; columellar margin slightly curved and thickened; the callus dilated over the umbilical fissure.

Length $8.2 \mathrm{~mm}$, breadth $2.6 \mathrm{~mm}$ (figured type specimen).

Habit: On the surface of Mesolhuria parva (THÉL)-- deep-sea seacucumber.

Type locality: Tosa Bay, Shikoku. 


\section{Balcis peronellicola KURODA et $\mathrm{H}_{\mathrm{ABE}}$}

(P1. VI, Fig. 17)

1950 Balcis peronellicola KURODA et HABE, Illust. Cat. Jap. Sáells, No. 9, p. 60.

Length $4.3 \mathrm{~mm}$, breadth $1.5 \mathrm{~mm}$ (ifgured type specimen).

Distribution: Yura, Wakayama Pref. (type locality); Suiken in Wakayama City and Mukai-jima near Onomichi City, Hiroshima Pref., Honshu.

Habit: Around the mouth of Peronella japonica MoRTensen-a sanddwelling cake-urchin.

\section{Balcis auratus (S. HrRASE)}

(P1. VI, Fig. 11)

1920 Melanella aurata S. HIRASE, Zool. Mag. (Japan), 32 (385), p. 345, pl. 6.

1927 Melanella aurata S. HIRASE, Nippon Dobutsu.Zukan, p. 1462.

Length $4.0 \mathrm{~mm}$, breadth $1.5 \mathrm{~mm}$ (figured specimen collected from Shirahama, Wakayama Pref., Honshu).

Habit: On the shell of Anthocidaris crassispina (A. AGASsIz)-littoral sea-urchin.

Distribution: Naruto, Tokushima Pref. (Type locality) and Shirahama, Wakayama Pref., Honshu.

\section{Balcis langfordi (DALL)}

1925 Strombiformis langfordi DAIL, Nautilus, 38 (3), p. 97.

Habit: On the shell of Anthocidaris crassispina (A. Agassiz).

Type locality: Shimoda, Izu Peninsula, Honshu.

\section{Balcis komaii $\mathrm{H}_{\mathrm{ABE}}$}

(P1. VI, Fig. 10)

1950 Balcis komaii HABE, Illust. Cat. Jap. Shells, No. 6, p. 44.

Length $4.1 \mathrm{~mm}$, breadth $1.9 \mathrm{~mm}$ (figured type specimen).

Habit: On the arm of Comanthus japonicus (J. MULLER) -littoral crinoid.

Type locality: Shirahama, Wakayama Pref., Honshu. 


\section{Bal cis akauni new species}

(Pl. VI, Fig. 5)

Shell slender, acutely attenuate above, slightly bent to the left side backwardly, thin semitransparent and glassy; whoris 10 in number, each whorl flattened and suture appressed; apex small but blunt; varices impressed and marked with brown-colored stripes, one on each whorl, and they are all on the right side, where they form a stepped ascending line; body whorl rather large and high, a half of shell length, decorated with a narrow brown spiral dotted line at the periphery; aperture elongate oval; outer margin gently curved and arcuate forwardly; columeilar margin rather straight, thickened and dilated on the umbilical fissure.

Length $4.6 \mathrm{~mm}$, breadth $1.5 \mathrm{~mm}$, (figured type specimen).

Habit: On the shell of Pseudocentrotus depressus (A. Agassiz)-littoral sea-urchin.

Type locality: Shirahama, Wakayama Pref., Honshu.

Remarks: This new species may be distinguished from the allied species Balcis langfordi (DALI) in having the more slender shell and a dotted brown line at the periphery.

\section{Genus Stilapex IREDALE 1925}

\section{Stilapex philippinarum (SOWERBY)}

(PI. VI, Figs. 23, 24)

1900 Mucronalia philippinarum SOWERBX, Proc. Malac. Soc. London, 4, p. 127, pl. 11, fig. 5.

1944 Mucronalia mittrei HABE, Jap. Jour. Malac. (Venus), 13 (5-8), p. 192, fig. 14 (non Petit 1851)

1944 Mucronalia mittrei HABF, /. c., p. 312.

Height $10.4 \mathrm{~mm}$, breadth $5.3 \mathrm{~mm}$ (ifgured specimen collected from Shirahama, Wakayama Pref., Honshu).

Height $9.8 \mathrm{~mm}$, breadth $4.8 \mathrm{~mm}$ (figured specimen from the same locality).

Habit: On the peristome of Coscinasterias acuispina (STIMPSON) - littoral sea-star.

Distribution: Cebu, Philippines (type locality); Amami Islands; Shirahama, Wakayama Pref. and Sagami Bay, Honshu.

Remarks: The head of the animal of this species is white, except for the fleshy orange tentacles. The Siboga specimens obtained from the Pasir Pandjang, Binongka Island (Siboga Station No. 220) and so named, was parasi- 
tic on Heterocentrotus mammillatus (sea-urchin) and seems to be a distinct species closely allied to this species.

\section{Genus Mucronalia A. Adams 1860}

\section{Mucronalia lactea (A. ADAMS)}

(P1. VI, Fig. 15)

1863 Leiostraca (Mucronalia) lactea A. ADAMS, Jour. Linn. Soc, London, Zool., 7, p. 91.

1929 Mucronalia lactoa MAK.YAMA, Venus, 1 \{5), p. 168.

1944 Mucronalia lactea HABE, Jap. Jour. Malac. (Venus), 13 (5-8) p. 312.

1950 Mucronalia lactea KURODA et HABE, Illust. Cat. Jap. Shells, No. 9, p. 58, pl. 10, fig. 11.

Length $4.8 \mathrm{~mm}$, breadth $1.8 \mathrm{~mm}$ (figured specimen collected from Hatakejima in the Tanabe Bay, Wakayama Pref., Honshu).

Habit: In the coelom of Leptosynapta ooplax (MARENZELLAR)-sand-dwelling sea-cucumber.

Distribution: Tanabe Bay, Wakayama Pref. (tyre locality) and Mukaijima near Onomichi City, Hiroshima Pref., Honshu.

\section{Mucronalia exilis A. ADAMS}

(Pl. VI, Fig. 14)

1862 Mucronalia exilis A. ADAMS, Ann. Mag. Nat. Hist., (3), $9\{52\}$, p. 295.

1863 Laiostraca (Mucronalia) A. ADAMS, Jour. Linn. Soc. London, Zool., 7, p. 91.

1929 Mucronalia exilis MAKIYAMA, Venus, 1, p. 168.

1944 Mucronalia exilis HABE, Jap. Jour. Malac. (Venus), $13\{5-8\rangle$, p. 311.

1950 Mucronalia exilis KURODA et HABE, Illust. Cat. Jap. Shells, No. 9, p. 58, pI. 10, fig. 12.

Length $2.8 \mathrm{~mm}$, breadth $1.1 \mathrm{~mm}$ (figured specimen collected from Suiken, Wakayama City, Honshu).

Distribution: Shimonoseki, Yamaguchi Pref. (type locality); Suiken, Wakayama City and Tanabe Bay, Wakayama Pref., Honshu. 


\section{Family Stiliferidae \\ Genus Stilifer BRODERIP 1832 \\ 16. Stilifer ovoideus (H. et A. ADAMS)}

(Pl. VI, Fig. 18)

1850 Stylifer astericola H. A. ADAMS et REEVE, Zool. Voy. Samarang, Moll., p. 47, pl. 17, fig. 5 (non BRODERIP 1832)

1853 Stylifer ovoideus $\mathrm{H}$. et A. ADAMS, Gen. Rec. Moll., 1, p. 239, pl. 25, fig. 4.

1878 Stylifer ovoideus SOW ERBY, Conch. Icon., sp. 2.

1884 Stylifer ovoideus SOWERBY, Thes. Conch., 5, p. 159, pl. 479, fig. 7.

1886 Stylifer woideus TRYON, Man. Conch., 8, p. 290, pl. 71, fig. 39.

1901 Stilifer ovoideus Sow ERBY, Proc. Malac. Soc. London, 4, p. 209.

1901 Siylifer ovoidea Cl.ESSIN, Conch. Cab., 1 (28), p. 241, pl. 40, fig. 8.

1927 Stilifer celebensis S. HIRASE, Nippon Dobutsu.Zukan, p. 1462.

1932 Stilifer celebensis S. HIRASE, Proc. Malac. Soc. London, 20 (1), pp. 73-76, pls. 7, 8 (non KÜKENTHAL 1897).

1933 Stylifer celebensis KURODA, Venus, 4 (4), p. 208.

Length $7.4 \mathrm{~mm}$, breadth $5.3 \mathrm{~mm}$ (figured specimen collected from Tomioka, Amakusa Island, Kyushu).

Habit: In the arm of Certonardoa semiregularis (MÜLLER et $\mathrm{T}_{\mathrm{ROSCHEL}}$ ).

Distribution: Borneo (type locality); Tomioka, Amakusa, Kyushu; Kushimoto, Wakayama Pref., Misaki and Hayama, Kanagawa Pref., Honshu.

\section{Stilifer utinomii $\mathrm{H}_{\mathrm{ABE}}$ (emend.)}

(P1. VI, Fig. 22)

1951 Silififer utinoumii (sic) HABE, Illust. Cat. Jap. Shells, No. 14, p. 93 (a typographical error)

Length $8.9 \mathrm{~mm}$, breadth $5.0 \mathrm{~mm}$ (figured type specimen).

Habit: In the arm of Linckia guildingii GRAY-Deep-sea sea-star.

Type locality: Shirahama, Wakayama Pref., Honshu.

\section{Stilifer ophidiastericola $\mathrm{HABE}$}

(P1. VI, Fig. 19)

1951 Siilifer ophidiastericola HABE, Illust. Cat. Jap. Shells, No. 14, p. 94.

Length $3.5 \mathrm{~mm}$, breadth $2.2 \mathrm{~mm}$ (figured type specimen). 
Habit: In the arm of Ophidiaster cribrarium LüTKEN and Ophidiaster loliori FISHER.

Distribution: Tomioka, Amakusa, Kyushu (type locality) and Kushimoto, Wakayama Pref., Honshu.

\section{Genus Rosenia Schepman 1913}

\section{Rosenia castanea (DALL)}

(P1. VI, Figs. 7, 8)

1925 Stilifer castanea DAT.L, Nautilus, 38 (3), p. 97.

1925 Stilifer pardepressus DALI, l. c., p. 97.

1929 Stilifer castaneus Is. TAKI, Venus, 1 (4), p 116, fig. 58.

1943 Littorina-like form MoRTENSFn, Monogr. Echinoidea, 3 (1), Camarodonta 1, p. 545.

Length $3.5 \mathrm{~mm}$, breadth $3.1 \mathrm{~mm}$ (figured specimen collected from Emi, Chiba Pref., Honshu).

Habit: On the shell of Pseudocentrotus depressus (A. Agassiz)-littoral sea-urchin.

Distribution: Emi and Chikura, Chiba Pref.; Kurosaki, Miura Peninsula, Kanagawa Pref. and Goza, Mie Pref., Honshu.

\section{Rosenia yamamotoi new species}

(P1. VI, Fig. 21)

Shell small, ovate, yellowish white, smooth and polished, with weak growth lines; whorls 4 in number, of which two nuclear whoris are obliquely mucronate; body whorl very large, roundly inflated, separated from the penultimate whorl by the distinctly marginated suture; aperture wide; outer margin arched, thin and sharp; columellar margin also curved, slightly thickened and dilated; umbilicus not perforated. No operculum.

Length $2.2 \mathrm{~mm}$, breadth $1.7 \mathrm{~mm}$ (figured type specimen).

Habit: On the shell of young specimen of Pseudocentrotus depressus (A. AgASSIZ).

Type locality: Shisô. jima, off Shirahama, Wakayama Pref., Honshu.

Remarks: This new species closely resembles Stilifer sibogae ScHEPMANN $1909^{7}$ from the East Indies, but it may be distinguished from the latter in the obliquely mucronate apex and less globular body whorl.

7) Siboga Exped. Monogr. 49 (2), p. 5 ; l. c., 49 (1), p. 241. 


\section{Rosenia tokiokai n€w species}

(P1. VI, Figs. 3, 12, 13)

1940 Mucronalia (?) sp. MoRTENSEN, Monogr. Echinoidea, 3 (1), Aurodonta, p. 251, pl. 65, figs. 2,3 .

Shell ovate, semitransparent, milky white, smooth and polished; apex pointed as in Stilifer and coloured snowy white; whorls 9 in number, each whorl somewhat convex, suture considerably impressed; body whorl large. rounded, being contained a half of the shell lensth; aperture ovate; outer margin arcuate, arched forwardly; columellar margin also curved, somewhat thickened; umbilicus closed. No operculum.

Length $8.6 \mathrm{~mm}$, breadth $5.2 \mathrm{~mm}$ (Egured type specimen).

Habit: On the peristome of Diadema seiosum (GRAY)-littoral sea-urchin.

Type locality: Shirahama, Wakayama Pref., Honshu.

Remarks: This new species is allied to Stilifer mitirei PETiT (1851) ${ }^{8,9}$ found on a Hawaiian Diadema, but the latter has less pointed early whorls in the young stage.

\section{Genus Kiramodulus Kuroda 1949}

\section{Kiramodulus lacteus KURODA}

(PI. VI, Figs. 1, 2)

1949 Kiranodulus lacteus KURODA, Jap. Jour. Malac. (Venus\}, 15 (5-8), pp. 72-75, fig. 1.

Length $6.3 \mathrm{~mm}$, breadth $7.6 \mathrm{~mm}$ (figured type specimen).

Habit: On the arm of a deep-sea sea-star, probably belonging to Paragonaster or Perissogonasier (according to the identification by Dr. R. HAYASHI).

Type locality: Tosa Bay, Shikoku.

Remarks: The systematic position of this genus and species was quite obscure at its establishment, but later the original author (1950) ${ }^{10}$ noticed the fact that the protoconch of this species is Stilifer-like, quite characteristic in gastropods. I also could confirm that the animal of this species has no radula. Thus I think that this genus should be placed in the family Stiliferidae.

8) Jour. de Conchyl, 2, p. 27, pl. 2 , figs. $8,9$.

9) Pilsbry 1920, Proc. Acad. Nat. Sci. Phila., 72, p. 298, figs. 2a, b.

10) Yumehamaguri, no. 51, p. 79 , textfig. (a mimeographed journal). 


\section{Parasitic Gastropods Found in Echinoderms}

\section{Incertae sedis}

\section{3. "Eulima" sp.}

1921 Melanella sp. A. H. CtARK, Monogr. Exist. Crinoids, 1, Comatulids 2 (U.S. Nat. Mus., Bull. 82), p. 649

1927 Eulima sp. GIsLÉn, Vid. Medd. Dansk Naturh. Foren. Köbenhavn, 83, p. 40.

1950 Eulima sp. A. H. CLARK, Monogr. Exist. Crinoids, 1, Comatulids 4c, p. 20.

Habit: On the arm of Stenometra diadema: (A. H. CLARK).

Distribution: Off Kyushu (lat. $32^{\circ} 17^{\prime \prime N}$., long. 128 $11^{\prime \prime E}$.), $201 \mathrm{~m}$; southern Japan, from 80 to 170 fathoms.

\section{4. "Eulima” sp.}

1922 Eulima sp. GTSI'́n, Nova Acta Reg. Soc. Sci. Upsaliensis, (4) 5, p. 94.

1947 Eulima (probably Sabinella or Metanella) sp. A. H. CLARK, Monogr. Exist. Crinoids, 1, Comatulids $4 b$, p. 373 .

Habit: On the arm of Neometra multicolor (A. H. CLARK).

Locality: Goto Islands, Kyushu, $164 \mathrm{~m}$.

\section{5. "Macronalia" sp.}

1943 Mucronalia (?) sp. MORTENSEN, Monogr. Echinoidea, 3 (2), Camarodonta 1, p. 251.

Habit: On the young specimen of Temnotrema sculpium A. AgAssiz.

Locality: Mutsu Bay, Honshu.

\section{6. "Mucronalia" sp.}

1943 Mucronalia (?) sp. MORTENSEN, l. c., p. 251.

Habit: On Temnotrema sculpium A. Acassiz.

Locality: Off Okino-shima, Kyushu.

$$
\text { 27. "Stilifer" sp. }
$$

1917 Siylifer-like sp. MATSUMOTO, Monograph of Japanese Ophiuroidea (Journ. Coll. Sci. Imp. Univ. Tokyo, 38, p. 222.

Habit: At the genital slits of Ophiothrix macrobrachia CLARK.

Locality: Sagami Sea and Uraga Channel. 


\section{Family PaEdorhoropidae \\ Genus Paedophoropus IwaNOFF 1933}

28. Paedophoropus dicoelobius IWANOFF

1933 Paedophoropus dicoelobius IWANOFF, Zool. Anz., 104, pp. 161-165.

Habit: In the polian vesicle of Eupyrgus pacificus ÖSTERGREN-deep-sea sea-cucumber.

Locality: Japan.

\section{Family Asterophilidae}

Genus Asterophila Randall et Heath 1912

29. Asterophila japonica RANDALL et HEATH

1912 Asterophila japonica RANDAIl et HEATh, Biol. Bull., 22, pp. 98-103, pls. 1 \& 2.

Habit: In the coelom of the arm of Pedicellaster $=P$. magister orientalis FISHER) - sea-star.

Locality: Off the coast of Corea, 150-163 fathoms. (Albatross Stations 4861-4867). 


\section{EXPLANATION OF PLATE VI}

(Each figure is unequally magnified.)

Figs. 1,2. Kiramodulus lacteus KURODA (type specimen) $(6.3 \mathrm{~mm} \times 7.6 \mathrm{~mm})$

3. Rosenia tokiokai n. $\mathrm{sp}$. $(6.0 \mathrm{~mm} \times 3.6 \mathrm{~mm})$

4. Balcis robustus (A. ADAMs) $(4.8 \mathrm{~mm} \times 2.4 \mathrm{~mm}$ )

5. Balcis akawni n. sp. (type specimen) $(4.6 \mathrm{~mm} \times 1.5 \mathrm{~mm})$

6,9. Balcis yamazii n. sp. (type specimen) $(7.5 \mathrm{~mm} \times 3.1 \mathrm{~mm}$ )

7. Rosenia castanea (DALL) $(3.5 \mathrm{~mm} \times 3.1 \mathrm{~mm})$

8. Rosenia castanea (DALL) (perdepressus-form) $(2.8 \mathrm{~mm} \times 2.8 \mathrm{~mm}$ )

10. Balcis komaii $\mathrm{HABE}$ (type specimen) $(4.1 \mathrm{~mm} \times 1.9 \mathrm{~mm}$ )

11. Balcis auratus (S. HIRASE) $(4.0 \mathrm{~mm} \times 15 \mathrm{~mm}$ )

12,13. Rosenia tokiokai $\mathrm{n}$. $\mathrm{sp}$. (type specimen) $(8.6 \mathrm{~mm} \times 5.2 \mathrm{~mm}$ )

14. Mucronalia exilis (A. Adams $(28 \mathrm{~mm} \times 1.1 \mathrm{~mm}$ )

15. Mucronalia lactea (A. ADAMS) $(4.8 \mathrm{~mm} \times 1.8 \mathrm{~mm}$ )

16. Balcis astropecienicola KURODA et HABE (type specimen) $(7.2 \mathrm{~mm} \times$ $2.4 \mathrm{~mm}$ )

17. Balcis peronellicola KuRoda et HABE (type specimen) (4.3 $\mathrm{mm} \times$ $1.5 \mathrm{~mm}$ )

18. Stilifer ovoideus (H. et A. ADAMS) $(7.4 \mathrm{~mm} \times 5.3 \mathrm{~mm}$ )

19. Stilifer ophidiastericola $\mathrm{HABE}$ (type specimen) $(3.5 \mathrm{~mm} \times 2.2 \mathrm{~mm})$

20. Balcis kuronamako n. sp. (type specimen) $(8.3 \mathrm{~mm} \times 2.8 \mathrm{~mm})$

21. Rosenia yamamotoi n. $\mathrm{sp}$. (type specimen) $(2.2 \mathrm{~mm} \times 1.7 \mathrm{~mm}$ )

22. Stilifer utinomii HABE (type specimen) $(8.9 \mathrm{~mm} \times 5.0 \mathrm{~mm}$ )

23. Stilapex philippinarum (SowERBY) $(10.4 \mathrm{~mm} \times 5.3 \mathrm{~mm}$ )

24. Stilapex philippinarum (SOWERBY) $(9.8 \mathrm{~mm} \times 4.8 \mathrm{~mm}$ )

25. Balcis soliduloides HABE (type specimen) $(7.5 \mathrm{~mm} \times 2.8 \mathrm{~mm}$ )

26, 27. Balcis teramachii n. $\mathrm{sp}$. (type specimen) $(8.2 \mathrm{~mm} \times 2.6 \mathrm{~mm}$ ) 
Publ. Seto Mar. Biol. Lab., II, 2 (1952) PLÄT̄E VI.
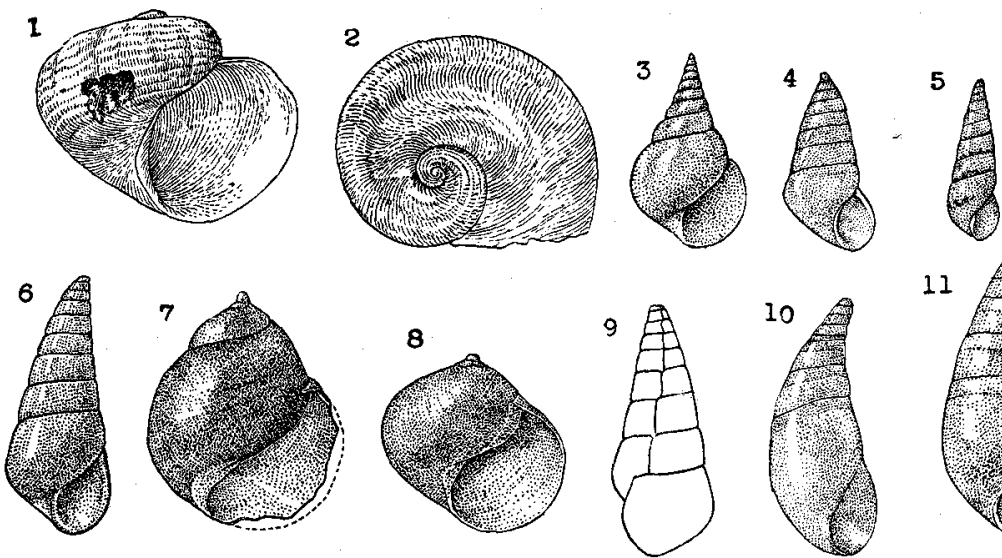

11
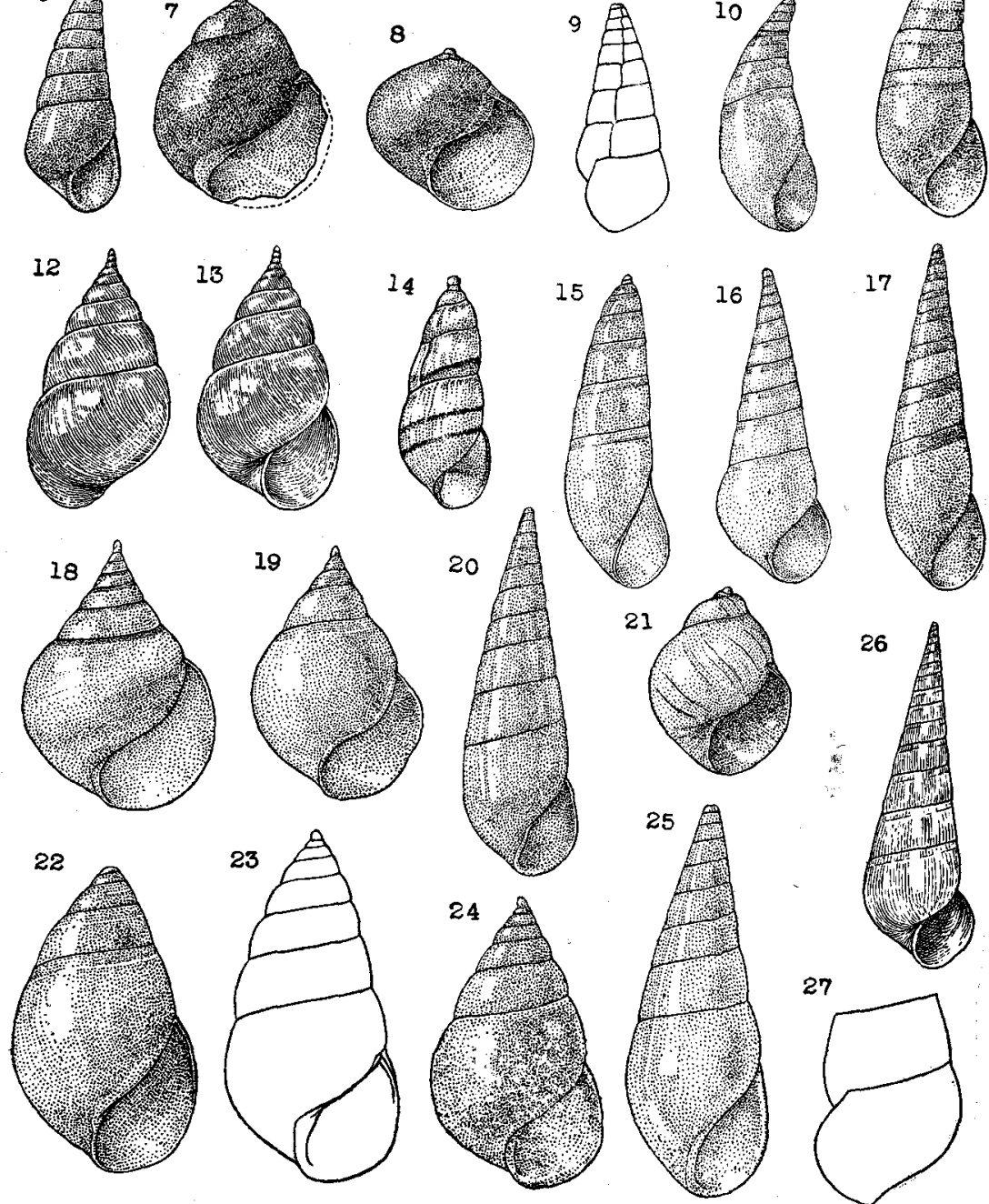

T. Habe: Parasitic Gastropods Found in Echinoderys. 